\title{
Banque d'instruments de mesure en recherche : Une innovation au service des membres chercheurs en sciences infirmières
}

\author{
Sylvie Le May', Marilou Bourque, Michelle Proulx, Matthias Duc
}

Résumé : Introduction : Face aux difficultés que rencontrent ses enseignants et étudiants à retrouver des instruments de mesure valides dans les bases de données, le Réseau de Recherche en Interventions en Sciences Infirmières du Québec (RRISIQ) a récemment choisi de développer une banque d'instruments de mesure accessible et bien documentée utilisant le logiciel bibliographique Zotero. Cet article a pour but de décrire la Banque d'instruments du RRISIQ, d'en exposer les défis et ses perspectives de développement. Description : La Banque comprend plus de 1400 liens ou références à des instruments de mesure reliés aux interventions cliniques, à l'organisation des services infirmiers et à la formation infirmière. L'utilisateur a accès à des références bibliographiques d'articles scientifiques sur les instruments, en anglais et en français. En naviguant dans la Banque, il clique sur l'article de son choix, obtenant ainsi une description bibliographique complète, dont une adresse web lui permettant d'accéder en ligne au plein texte. Résultats : La Banque d'instruments Zotero nécessite un faible coût d'entretien technique pour effectuer des sauvegardes, résoudre les difficultés et gérer les demandes d'accès. Elle est appréciée par ses utilisateurs. Discussion : La Banque prendra de l'ampleur dans les années à venir et des démarches sont actuellement réalisées par l'équipe pour la publiciser davantage auprès de ses membres et de leurs étudiants. L'équipe envisage de la rendre disponible à d'autres équipes de recherche du Québec.

\section{Introduction}

La navigation et la localisation de matériels pertinents et précis dans les grandes Banques de données représentent un réel défi, tant pour les étudiants des programmes universitaires, les infirmières que pour les professeurs $[1,2,3]$. Ce défi prend d'autant plus d'importance que les milieux académiques et cliniques ont accès à un nombre important de ressources informationnelles, sans toujours disposer des connaissances nécessaires pour réaliser des recherches ciblées $[4,5,6]$. Il appert que les enseignants et les étudiants des milieux académiques s'en tiennent, le plus souvent, à un nombre limité de bases de données avec lesquelles ils sont familiers, et qu'ils ne semblent pas bien connaître l'éventail des ressources documentaires qui s'offrent à eux.
Le Réseau de Recherche en Interventions en Sciences Infirmières du Québec (RRISIQ) existe depuis 2012 et comprend 169 membres chercheurs en sciences infirmières et leurs étudiants. Le Réseau a récemment choisi de mettre en place, pour ses membres et leurs étudiants, une Banque d'instruments de mesure et de guides d'entrevue facilement accessible et bien documentée. Une revue exhaustive de la littérature n'avait pas permis de retrouver de banques similaires. L'objectif visé par les auteurs était de rendre disponible une ressource documentaire facilement accessible et conviviale pour les membres du Réseau.

Cet article a pour but de décrire la Banque d'instruments du RRISIQ, d'exposer les défis rencontrés ainsi que les perspectives de développement.

Sylvie Le May. Centre de recherche du CHU Sainte-Justine, 3175 Côte-Ste-Catherine, Montréal, QC H3T 1C5

Marilou Bourque. Direction des Bibliothèques, Université de Montréal

Michelle Proulx. Centre de recherche du CHU Sainte-Justine, 3175 Côte-Ste-Catherine, Montréal, QC H3T 1C5

Matthias Duc. Direction des Bibliothèques, Campus Saint-Jean, Université de l'Alberta

${ }^{1}$ L'auteur principal (courriel: sylvie.lemay@umontreal.ca)

Cet article a été critiqué par des pairs. La rédactrice invitée : Tara Landry, Hôpital général de Montréal. 


\section{Description}

La création de la Banque a débuté en janvier 2013 par une analyse des besoins effectuée auprès des membres via un sondage avec l'application SurveyMonkey. L'équipe souhaitait connaître les préoccupations et les besoins des membres en ce qui concerne les types d'instruments et les grilles d'entrevue que devait offrir la Banque.

Parallèlement, des démarches ont été effectuées pour identifier le support le plus approprié pour héberger la Banque. L'équipe a opté pour l'utilisation d'un logiciel bibliographique existant déjà plutôt que de programmer un nouvel outil. Au nombre des logiciels les plus utilisés pour la gestion documentaire (EndNote, Reference Manager, RefWorks, Zotero, Mendeley, BibTeX), EndNote et Zotero étaient les plus conviviaux. Les auteurs ont opté pour le logiciel Zotero en raison de sa flexibilité au niveau de ses fonctionnalités de partage et parce qu'il est simple d'utilisation, gratuit et accessible à tous.

\section{Déploiement de la Banque}

Plusieurs éléments ont guidé le déploiement de la Banque dans Zotero. L'équipe a opté pour un thésaurus unilingue anglophone. Ce choix s'est imposé alors que la majorité des bases de données et des thésaurus utilisés en sciences infirmières sont de langue anglaise, et que la tâche d'offrir un outil bilingue aurait été trop importante sans être indispensable, considérant que les membres du RRISIQ travaillent généralement avec des outils rédigés en anglais. Suite à une consultation menée auprès des chercheurs du Réseau les invitant à nommer les sources qu'ils utilisaient le plus souvent pour la recherche de documentation, les auteurs ont privilégié le recours au thésaurus de CINAHL. Reconnu pour sa qualité et sa grande couverture du domaine des sciences infirmières, le thésaurus de CINAHL permettait le mieux de décrire les trois domaines de recherche couverts par le Réseau (expertise clinique, administration des services infirmiers et formation infirmière).

D'autre part, des démarches ont été entreprises pour s'assurer du respect du droit d'auteur. Après avoir consulté Copibec (http://www.copibec.qc.ca), un organisme de gestion des droits d'auteurs au Québec, et la direction des bibliothèques de l'Université de Montréal, il a été établi que la Banque ne pouvait contenir le plein texte des documents sélectionnés. Néanmoins, une solution a été trouvée afin de contourner cet obstacle et de permettre d'accéder au plein texte lorsque souhaité : un lien URL est inclus dans la référence bibliographique. Cette adresse permet aux usagers d'accéder à la ressource en ligne à condition qu'ils se soient authentifiés (via leur code d'utilisateur et mot de passe) et à condition que l'institution à laquelle ils sont rattachés possède une licence d'utilisation de la ressource documentaire choisie. Si l'adresse URL proposée provient de l'Université de Montréal et donnera à cette communauté spécifique un lien instantané à la ressource, les fournisseurs de bases de données disposent de résolveurs de liens permettant dans une majorité de cas de reconnaître un usager d'une autre institution et de vérifier si celle-ci a acquis une licence d'utilisation de la ressource documentaire. L'usager est donc reconduit vers la ressource après avoir été « reconnu " (ainsi que l'institution à laquelle il est affilié) par le fournisseur de la base de données.

Enfin, il fallait s'assurer que l'outil Zotero comporte un espace de stockage suffisant pour les besoins de la Banque, ce qui s'est avéré. Le logiciel offre 300Mo d'espace gratuit, et la possibilité de payer à moindre coût pour obtenir de l'espace supplémentaire selon GMU. Il offre aussi la possibilité de stocker les données sur un serveur externe via le protocole WebDAV (Gestion de fichiers avec serveurs distants). Cependant, après analyse, cette option n'est pas apparue possible dans le cas d'un groupe partagé.

L'accessibilité à la Banque d'instruments Zotero a initialement été vérifiée auprès de six membres du RRISIQ provenant de différentes universités affiliées à partir d'une base de données pilote, ce qui a permis d'améliorer sa structure, de confirmer certains besoins et de procéder au téléchargement de références. Pour ce faire, les membres du Réseau ont de nouveau été consultés pour identifier les thèmes ou concepts dans leur domaine de recherche respectif pour lesquels ils souhaitaient que la Banque fournisse de la documentation (ex. sécurité des patients, évaluation de la douleur, transfert des connaissances). Un plan de concepts par domaine a été établi à partir desquels la recherche d'instruments et de grilles d'entrevue a été effectuée par l'interrogation des bases de données CINAHL, PubMed et Medline. La Banque a été publicisée sur le site du RRISIQ et rendue disponible aux membres au mois d'août 2013. 


\section{Structure et fonctionnement de la Banque}

Les membres du RRISIQ peuvent accéder à la Banque Zotero de deux façons : soit directement en ligne sur le site de Zotero, soit en téléchargeant le logiciel sur leur poste de travail à l'aide de l'extension Firefox ou en version « standalone » pour utilisation avec d'autres navigateurs. Comme il s'agit d'une base de données privée, le membre qui désire avoir accès à la Banque doit d'abord se créer un compte Zotero et ensuite faire une demande d'accès au bibliothécaire gestionnaire de la Banque. Celui-ci lui fait parvenir un message d'invitation à partir de l'interface de gestion des membres du groupe dans Zotero. Le membre n'a qu'à accepter l'invitation pour ensuite pouvoir consulter la Banque.

L'utilisateur de la Banque dispose d'un mode de recherche par dossiers et sous-dossiers (Figure 1). Plus précisément, il a accès à des dossiers par type de documents (articles théoriques, grilles d'entrevue, instruments de mesure) et à un dossier global pour chacun des trois domaines du RRISIQ (interventions cliniques, formation et gestion des soins infirmiers). Dans chacun des dossiers, un sous-dossier réunit les documents en français, ce qui correspond à l'un des besoins exprimés par certains des chercheurs du RRISIQ. L'utilisateur a aussi accès à un dossier Aide qui comprend des guides et aide-mémoires pour utiliser Zotero. Il peut aussi recourir à deux autres modes de recherche, soit à un nuage de mots-clés et à une boîte de recherche par auteurs et titres.

En navigant dans la Banque, le membre peut cliquer sur l'article de son choix et faire afficher sa description bibliographique complète (titre, auteur(s), type de document, résumé, etc.), de même qu'un lien URL lorsque disponible. Enfin, un champ Note fournit des indications sur le but visé par le document (par exemple, le développement et la validation d'un instrument), sur le nom de l'instrument lorsque possible (il arrive que des chercheurs ne donnent aucune appellation à leur instrument) et réfère l'utilisateur à des documents liés, si tel est le cas.
Les documents retenus réunissent des articles scientifiques traitant d'instruments de mesure et décrivant leur développement et (ou) leurs propriétés psychométriques. Ils regroupent également des articles qui permettent de juger de la pertinence de grilles d'entrevue (ex. liste de vérification ou Checklist en anglais, grille de questions ouvertes) avec à l'appui des données de recherche ou d'informations sur le développement des grilles. De même, ils peuvent contenir des articles théoriques faisant la revue d'instruments de mesure disponibles en rapport à un thème donné ou présentant des contenus théoriques en lien avec des aspects reliés aux qualités psychométriques d'instruments ou en appui au développement de grilles d'entrevues. Les documents sont liés entre eux, permettant soit de rediriger le lecteur d'un document (par exemple, un instrument de mesure) qu'il a ouvert vers un autre document (par exemples, article qui traite de l'instrument, un article théorique sur le thème de l'instrument). Pour être retenus et indexés, les articles doivent obligatoirement comporter l'ensemble des items de l'instrument à l'étude ou de la grille dont ils traitent, sauf exception. Un article qui ne présente pas l'instrument ou la grille dans son entièreté peut être indexé à la seule condition d'être relié à l'instrument ou à la grille dont il fait état dans un autre document à part.

Chaque document de la Banque est indexé par la professionnelle de recherche selon une liste de motsclés (section Marqueurs de Zotero) provenant des thésaurus mentionnés précédemment. La professionnelle y consigne les mots-clés décrivant le mieux le contenu du document (ex. thème principal, provenance de l'instrument, nom de l'instrument, caractéristiques du document), en ayant le souci d'inscrire les mots, French Translation, lorsque le document original est écrit en français. Une fois la fiche complétée, le document est classé par la professionnelle de recherche dans le ou les différents dossiers généraux et spécifiques lui correspondant. Cette dernière est également responsable de la mise à jour de la Banque. 
Fig. 1 Capture d'écran de la Banque d'instruments sous Zotero - Dossier général Instruments de mesure



\section{Résultats}

La Banque d'instruments Zotero contient à ce jour plus de 1400 références de documents abordant les trois domaines d'intérêts du RRISIQ. Elle nécessite un faible coût d'entretien technique (quelques heures par mois) pour effectuer des sauvegardes, résoudre les difficultés s'il y a lieu et gérer les demandes d'accès. Il est à noter que des sauvegardes sont effectuées automatiquement par Zotero et que par prudence, une sauvegarde supplémentaire est réalisée manuellement tous les deux mois et enregistrée dans un service Web de dépôt de documents. Seules quelques difficultés techniques ont été rencontrées jusqu'à maintenant, dont des problèmes de synchronisation entre le serveur de Zotero et sa version locale, lesquels ont été rapidement et facilement résolues.

La Banque a reçu une évaluation très positive de la part d'évaluateurs externes d'un organisme subventionnaire québécois dans le cadre de la demande de renouvellement du RRISIQ. Les évaluateurs ont souligné l'originalité, la pertinence et le travail rigoureux associé au développement de la Banque. De plus, il ressort qu'elle suscite de l'intérêt chez des chercheurs externes au RRISIQ. En effet, l'équipe du projet a récemment été approchée par une équipe en pédopsychiatrie pour leur fournir de l'information sur la Banque et éventuellement offrir des conseils pour le développement d'une Banque du même type.

De plus, la Banque semble être appréciée par ses utilisateurs, comme le montre un récent sondage sur son utilisation, administré auprès des membres du RRISIQ. Sur un total de 16 répondants, $23 \%$ des gens ayant utilisé la Banque ont mentionné qu'elle était facile ou très facile d'utilisation. Ils ont eu recours à la ressource pour les aider à la préparation de demandes de subvention. Le sondage a cependant permis de cerner des améliorations devant être apportées à la Banque. En effet, un nombre important de répondants $(88 \%)$ ont indiqué qu'ils n'avaient pas utilisé la Banque parce qu'ils n'en connaissaient pas l'existence, ou parce qu'ils ne savaient pas comment y avoir accès et comment naviguer dans celle-ci. Certains ont précisé que, connaissant maintenant l'existence de la Banque, ils n'hésiteraient pas à l'utiliser ou ont dit apprécier de pouvoir compter sur ce type de ressources. Des démarches ont depuis été entreprises auprès de l'équipe de direction du RRISIQ. Le Réseau travaille présentement à publiciser la Banque sur une base régulière et à en faciliter l'accès et l'utilisation en offrant un soutien pour le téléchargement de Zotero à partir des postes de travail des membres. Un nouveau gestionnaire de la Banque a été engagé. Diplômé en 
bibliothéconomie, il a pour mandat de solliciter directement les membres pour leur offrir de les aider à télécharger Zotero et à créer un accès à la Banque via leur bureau de leur ordinateur. Il a créé des tutoriels en anglais et en français pour permettre aux membres qui le désirent de télécharger Zotero et d'établir par euxmêmes leur accès à la Banque. Ces tutoriels sont accessibles via le site Web du RRISIQ et servent de supports additionnels pour l'accès à la Banque. Cette approche plus personnalisée pour le marketing de la Banque a généré beaucoup d'intérêt et s'est avérée un moyen efficace d'augmenter la visibilité et l'accès à la Banque aux chercheurs, à leurs étudiants des cycles supérieurs ainsi qu'à leurs agents de recherche.

\section{Discussion}

La Banque d'instruments Zotero prendra de l'ampleur dans les années à venir. L'équipe du projet vise à ce que le nombre d'instruments de mesure et de grilles d'entrevue augmente et que la qualité des références soit reconnue par la communauté scientifique. L'équipe souhaite également augmenter le nombre d'utilisateurs, en s'associant des chercheurs externes de la francophonie. Elle poursuivra ainsi ses efforts pour capter et indexer le plus grand nombre possible d'instruments et de grilles d'entrevues en français du Québec et d'ailleurs. Le développement d'une telle Banque d'instruments est facile à réaliser dans de brefs délais. Cela nécessite peu de ressources si ce n'est l'expertise complémentaire de chercheurs, de professionnels en bibliothéconomie et en recherche. Cependant, l'efficacité d'une telle ressource repose également sur la mise à jour et l'entretien de celle-ci. Ces activités requièrent d'identifier un responsable central de la gestion de la Banque de même que des ressources financières pour rémunérer le personnel. À cet effet, l'appui du RRISIQ a été indispensable pour créer et maintenir les activités au niveau de la Banque. Les démarches pour augmenter la visibilité et l'accès, et la pertinence de la Banque pour ses membres doivent se poursuivre. Enfin, l'équipe entrevoit de développer la Banque pour y inclure éventuellement d'autres ressources documentaires pouvant être utiles pour la recherche en interventions infirmières.

\section{Références}

1. Dee C, Stanley, EE. Information-seeking behavior of nursing students and clinical nurses : implications for health science librarians. J. Med. Libr. Assoc. 2005; 93(2) : 213-222.

2. Oakleaf M, Millet, MS, Kraus, L. All together now : getting faculty, administrators, and staff engaged in information literacy assessment. Portal : Libraries and the Academy. 2011; 11(3) : 831852.

3. Wahoush O, Banfield L. Information literacy during entry to practice : information-seeking behaviors in student nurses and recent nurse graduates. Nurs Educ Today. 2014; 34(2) : 208213. doi : 10.1016/j.nedt.2013.04.009

4. Lialiou P, Mantas J. Online information seeking behaviour by nurses and physicians : a cross sectional study. Stud Health Technol Inform. 2016; 225 : 33-37. doi : 10.3233/978-1-61499$678-1-801$

5. Marshall, JG, Morgan, JC, Klem, ML, Thompson, CA, Wells, AL. The value of library and information services in nursing and patient care. Online J Issues Nurs. 2014;19(3) : 1-11. doi : 10.3912/OJIN.Vol19No03PPT02

6. Ginex PK, Hernandez M, Vrabel M. A clinical librarian-nursing partnership to bridge clinical practice and research in an oncology setting. Oncol Nurs Forum. 2016; 43(5) : 549-552. doi : 10.1188/16.ONF.549-552 